\title{
ECOLOGIE DE L'OMBRE COMMUN
}

\author{
H. PERSAT \\ Département de Biologie Animale et Zoologie \\ Université Cl.-Bernard \\ 43, Bd du 11 Novembre 1918 \\ 69621 VILLEURBANNE
}

(Extrait du rapport final de l'étude sur l'Ombre commun engagée par le Conseil Supérieur de la Pêche auprès du Département de Biologie Animale de l'Université Claude-Bernard, LYON I).

L'Ombre commun est un poisson initialement assez largement répandu en Europe centrale et en Scandinavie, mais on le rencontre également plus au Sud, le long des chaines montagneuses (fig. 1). Bien que les glaciations aient fortement influencé sa distribution et permis par exemple sa pénétration en Angleterre, son arrivée en Europe semble nettement préglaciaire. 


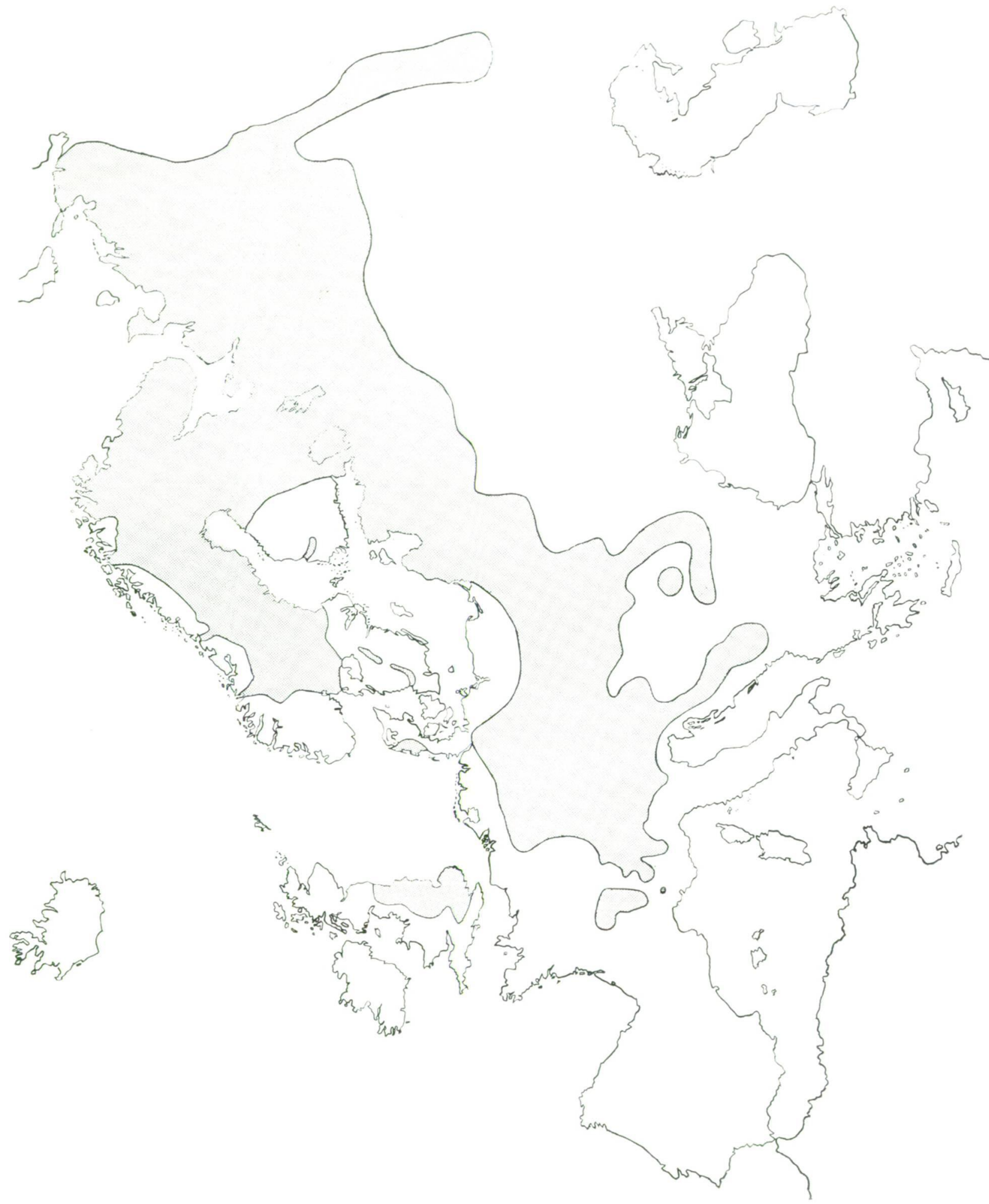

Figure 1 - Répartition géographique de l'Ombre commun en Europe. 
En France cette espèce n'existe à l'ètat naturel que dans certains cours d'eau des bassins du Rhin, du Rhône et de la Loire, à l'exclusion des bassins de la Seine et de la Garonne (fig. 2). L'Ombre a été introduit récemment dans de nombreuses autres rivières mais il semble éprouver des difficultés pour s'y maintenir, et l'on observe par ailleurs une forte diminution de ses populations dans son habitat d'origine (fig. 3). L'inefficacité des introductions peut être imputée soit à un manque d'études préalables des rivières choisies, entrainant le déversement d'alevins dans des eaux ne leur convenant pas, soit à une absence d'information suffisante auprès des pêcheurs qui, ne connaissant pas ce poisson, le capturent avant qu'il ne se reproduise, soit peut-être également à l'utilisation de souches trop nordiques non adaptées à nos rivières.

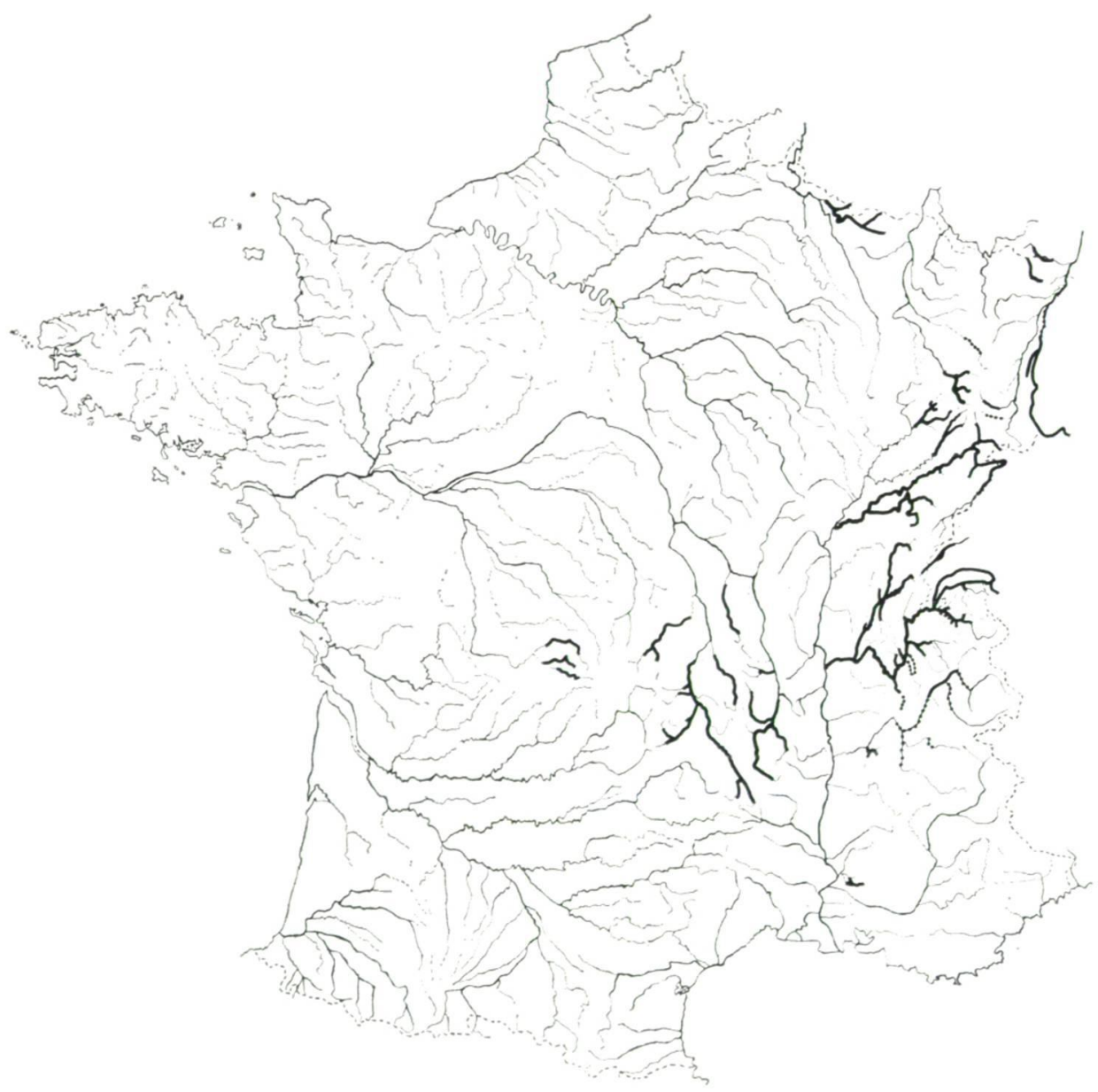

Figure 2 - Répartition géographique naturelle de l'Ombre commun en France vers 1900.

- En traits gras : habitat certain.

- En pointillés : habitat probable. 


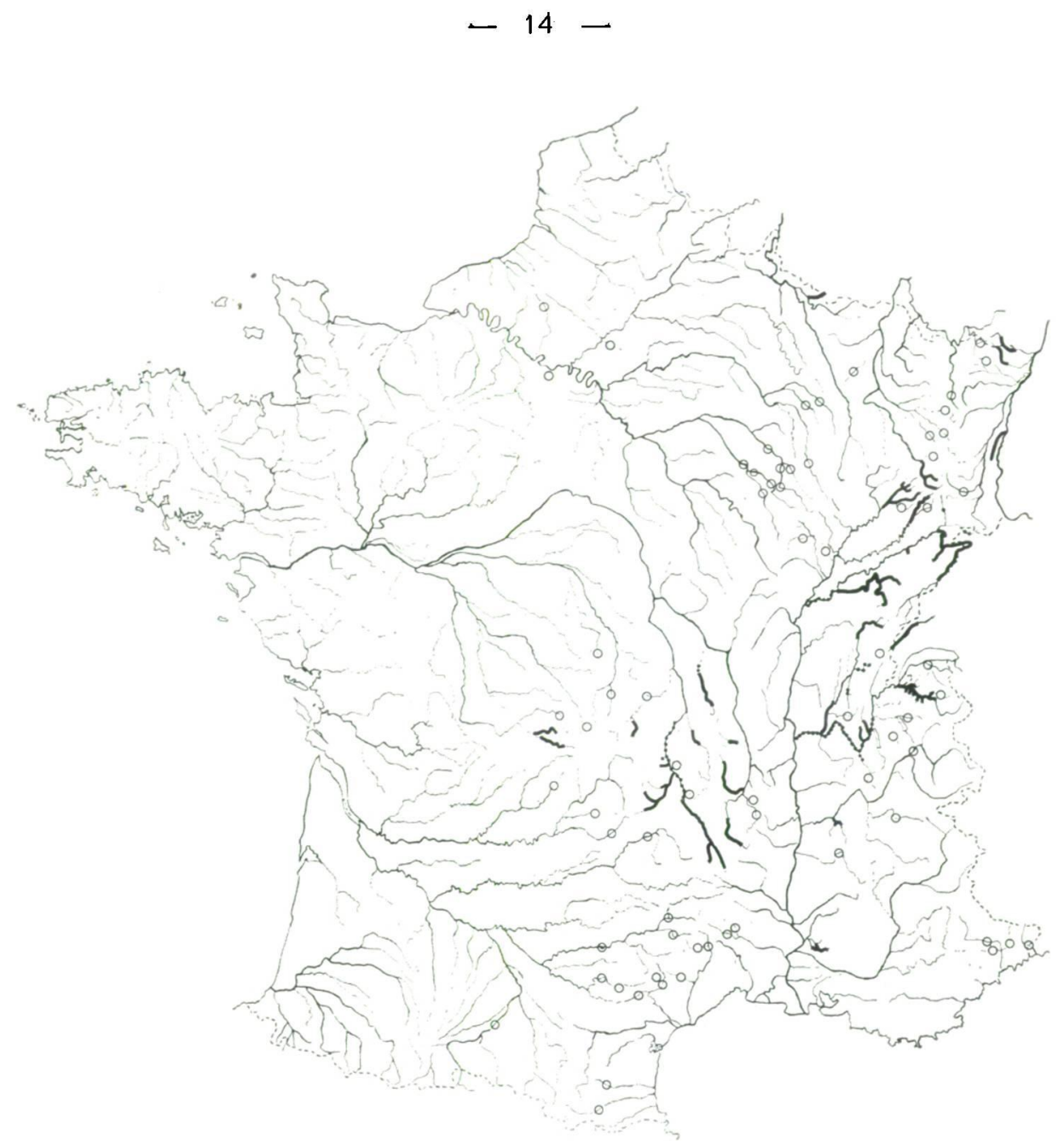

Figure 3 - Répartition géographique de l'Ombre commun en France en 1974.

- En traits gras : peuplements importants.

- En pointillés : peuplements en voie de disparition.

- Cercles : tentatives d'introduction ou de réintroduction.

Une analyse des conditions physicochimiques régnant dans son biotope a été réalisée dans deux rivières, l'Ain, rivière, du Jura calcaire, et l'Ance du Nord, rivière des monts granitiques du Forez, qui représentent les deux types principaux de rivières à Ombre de notre pays. Ces deux rivières présentent un certain nombre de caractères communs nécessaires à la survie de ce poisson :

- Pente modérée, de 1 à $8 \%$.

- Température moyenne estivale comprise entre 15 et $18^{\circ} \mathrm{C}$ avec des maximums instantanés n'atteignant jamais $24{ }^{\circ} \mathrm{C}$. 
- Oxygénation toujours proche de la saturation.

- Absence de pollution chronique mesurable.

- Gravières non colmatées susceptibles de servir de frayères.

Ces rivières diffèrent par un certain nombre de facteurs liés à la nature géologique de leurs bassins versants :

- $\mathrm{pH}$ variant de 6,6 dans l'Ance à 8,4 dans l'Ain.

- Conductivité s'échelonnant de 16 à près de $400 \mu \mathrm{mho} / \mathrm{cm}^{2} / \mathrm{cm}^{2}$.

- Concentration en calcium de 1 à $100 \mathrm{mg} / \mathrm{l}$.

Ces différences semblent bien tolérées par le poisson mais elles peuvent intervenir sur la production, la croissance et la densité de l'espèce.

L'examen de la résistance de jeunes Ombres de pisciculture à la température et au manque d'oxygène dissous montre que, au-dessus de $23^{\circ} \mathrm{C}$ dans une eau aérée, ou au-dessous de $5 \mathrm{mg} / \mathrm{l}$ d'oxygène dans une eau à $18-20^{\circ} \mathrm{C}$, l'Ombre commun se trouve en difficulté avec un pourcentage de pertes plus ou moins important dans la population; au-dessus de $25^{\circ} \mathrm{C}$ ou en-dessous de 4 $\mathrm{mg} / \mathrm{l}$, dans les mêmes conditions, tous les poissons meurent rapidement. Ces valeurs sont similaires à celles obtenues chez la plupart des autres espèces de Salmonidés.

Une étude comparative de la faune d'invertébrés benthiques des rivières et de contenus stomacaux d'Ombres communs révèle un régime assez varié suivant la faune disponible. Dans l'Ain, l'Ombre se nourrit essentiellement de Gammares, mais en proportion nettement inférieure à ce qui existe dans la rivière car il semble rechercher plutôt les insectes, principalement les subimagos d'Ephémères (Ephemerella et Baetis) dérivant à la surface et les larves de Trichoptères et de Diptères (Chironomides). Les jeunes Ombres de l'Ain évitent même complètement les Gammares. Dans l'Ance du Nord où les Gammares sont très rares, le régime de l'Ombre est encore plus diversifié, Ephémères, Trichoptères, Diptères, Coléoptères, Hyménoptères (fourmis), Mollusques..., et varie suivant l'abondance saisonnière des différentes proies. L'Ombre n'est donc habituement guère exigeant et semble assimiler ce qui se présente à sa portée, faune du fond et faune dérivante, pourvu que les proies soient suffisamment petites. Cependant, lorsque le choix est possible, les Gammares et les Plécoptères seraient moins appréciés, surtout des jeunes individus.

Nous avons étudié la croissance de l'Ombre commun dans la rivière d'Ain, et plus superficiellement dans l'Ance et la Loue. Cette croissance est extrêmement rapide (fig. 4) et atteint 15 et $29,5 \mathrm{~cm}$ respectivement à un et deux ans dans la rivière d'Ain. Cette croissance rapide semble cependant s'accompagner d'une longévité réduite (4 ou 5 ans).

La maturité génitale de l'espèce est atteinte dès l'âge de deux ans chez les plus grands mâles et les plus grandes femelles de la rivière d'Ain, et à trois ans chez tous les autres spécimens.

Le sexe des Ombres se détermine relativement aisément en comparant le développement de la partie postérieure de la nageoire dorsale, plus important chez le mâle (fig. 5). Pour plus de précision on peut mesurer la longueur de l'antépénultième rayon de la dorsale (l'avant avant-dernier) et la rapporter dans la fig. 6 à la longueur totale du poisson. Si le point obtenu est au-dessus de la droite le poisson sera un mâle; s'il est en-dessous ce sera une femelle; seuls 


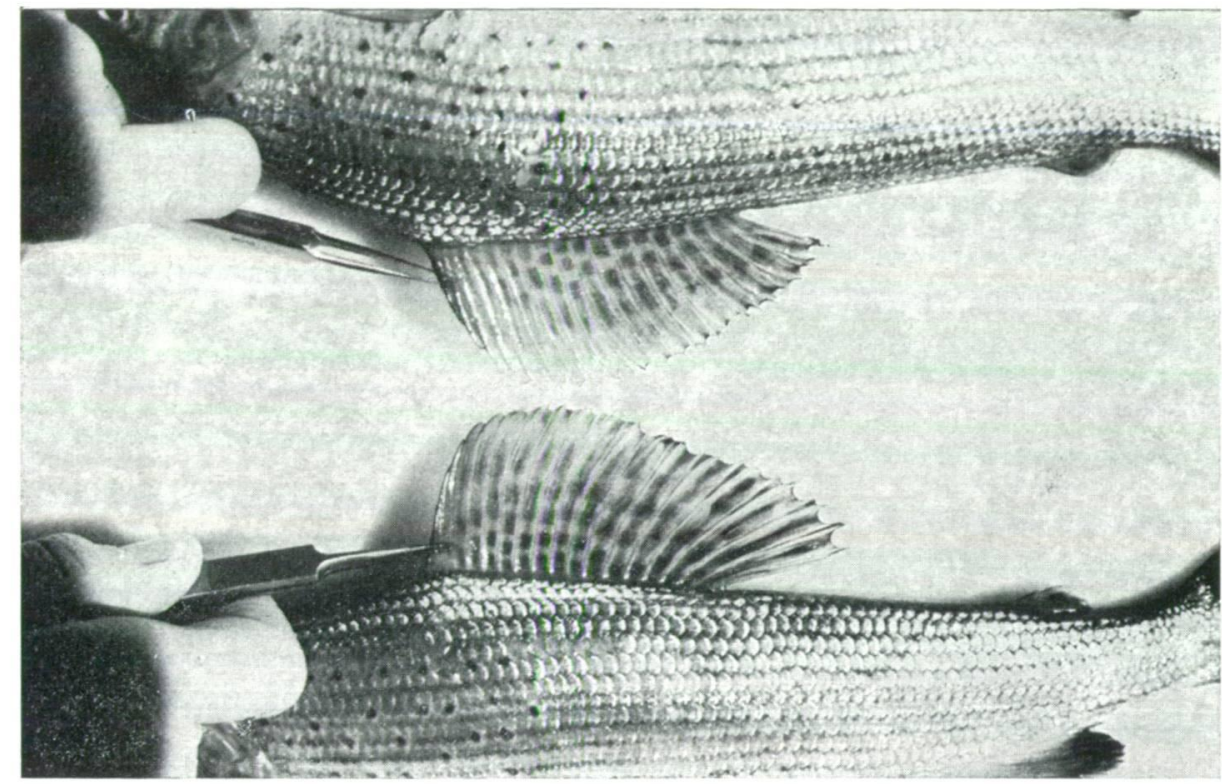

Figure 5 - Dimorphisme sexuel chez l'Ombre commun : le mâle (en bas) présente une nageoire dorsale beaucoup plus développée dans sa partie postérieure que celle de la femelle (en haut).

les points tombant à proximité immédiate de la droite ne permettront pas une détermination rigoureuse.

En ce qui concerne la taille légale de capture de cette espèce, si l'on tient compte des courbes de croissance de l'Ombre dans les rivières étudiées et des résultats de l'étude sur sa maturité, on aboutit aux constatations suivantes :

- A la taille actuelle de $27 \mathrm{~cm}$, les poissons sont protégés durant toute leur première année et seulement une partie de leur deuxième année, car leur taille moyenne à la fin de cette année est de $29 \mathrm{~cm}$.

La saison d'automne constitue actuellement un cap fatal à la plupart des Ombres de deux étés : selon les fiches de captures établies par les pêcheurs, près de $90 \%$ des poissons pêchés de septembre à décembre appartiennent à ce groupe d'âge.

Les individus croissant le plus vite auront le plus de chance d'être capturés, car ils atteindront la taille de $27 \mathrm{~cm}$ dès la fin d'août. Ceux croissant normalement commenceront à dépasser cette limite au mois d'octobre et ce sont finalement les poissons grandissant le plus lentement qui sont effectivement protégés. On aboutit donc involontairement à une sélection de fait des poissons les moins productifs.

Les poissons pêchés à l'automne de leur deuxième année ne se sont évidemment jamais reproduits et ceux susceptibles de se reproduire au printemps suivant; étant justement les plus grands, sont en grande partie capturés. 


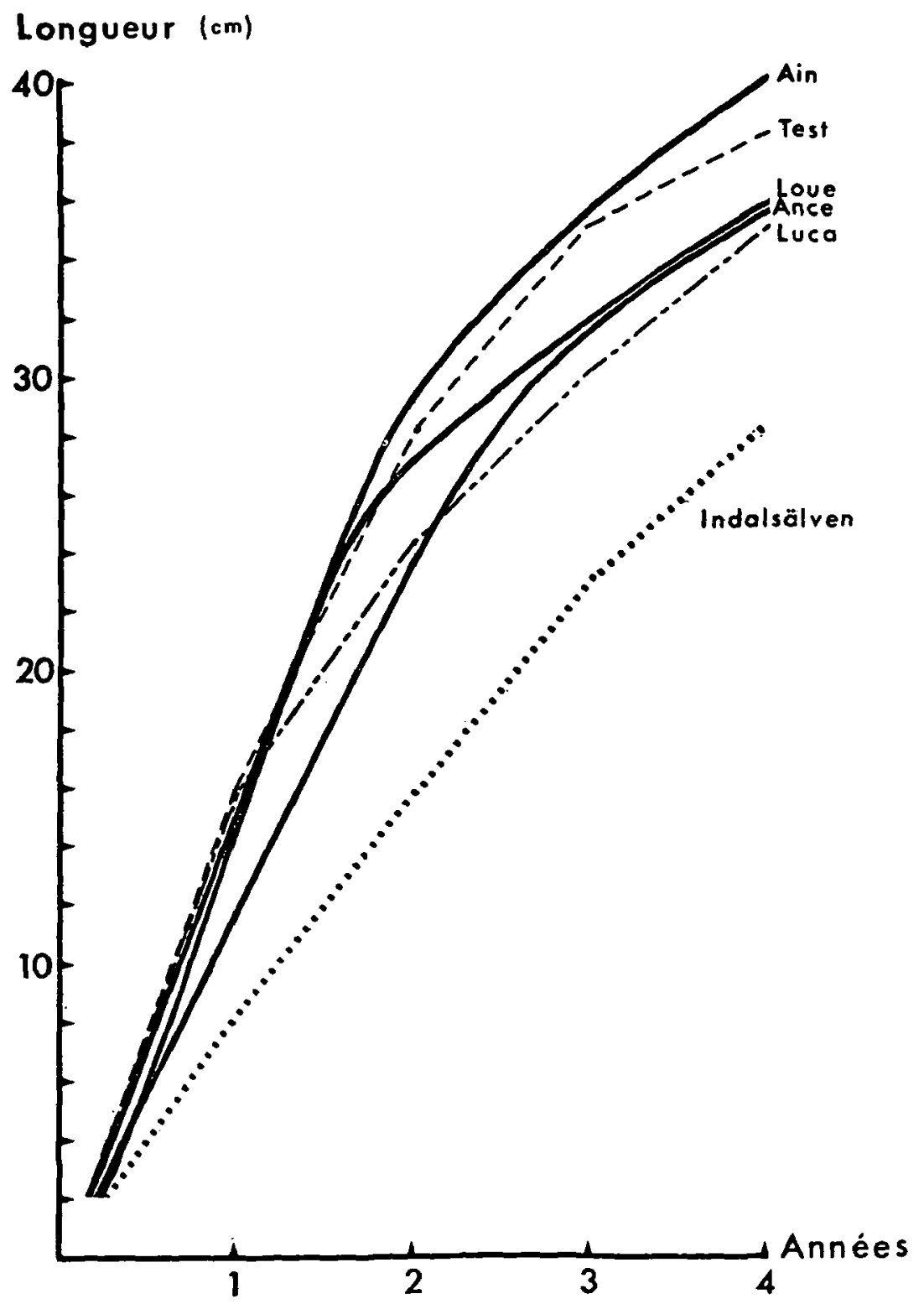

Figure 4 - Taux de croissance des Ombres de l'Ain, de la Loue, de l'Ance, de la Test (Grande-Bretagne), de la Luca (Yougoslavie) et de l'Indalsälven (Suède), les trois derniers selon respectivement : HUTTON 1923, IANKOVIC 1960 et PETERSON 1968, 


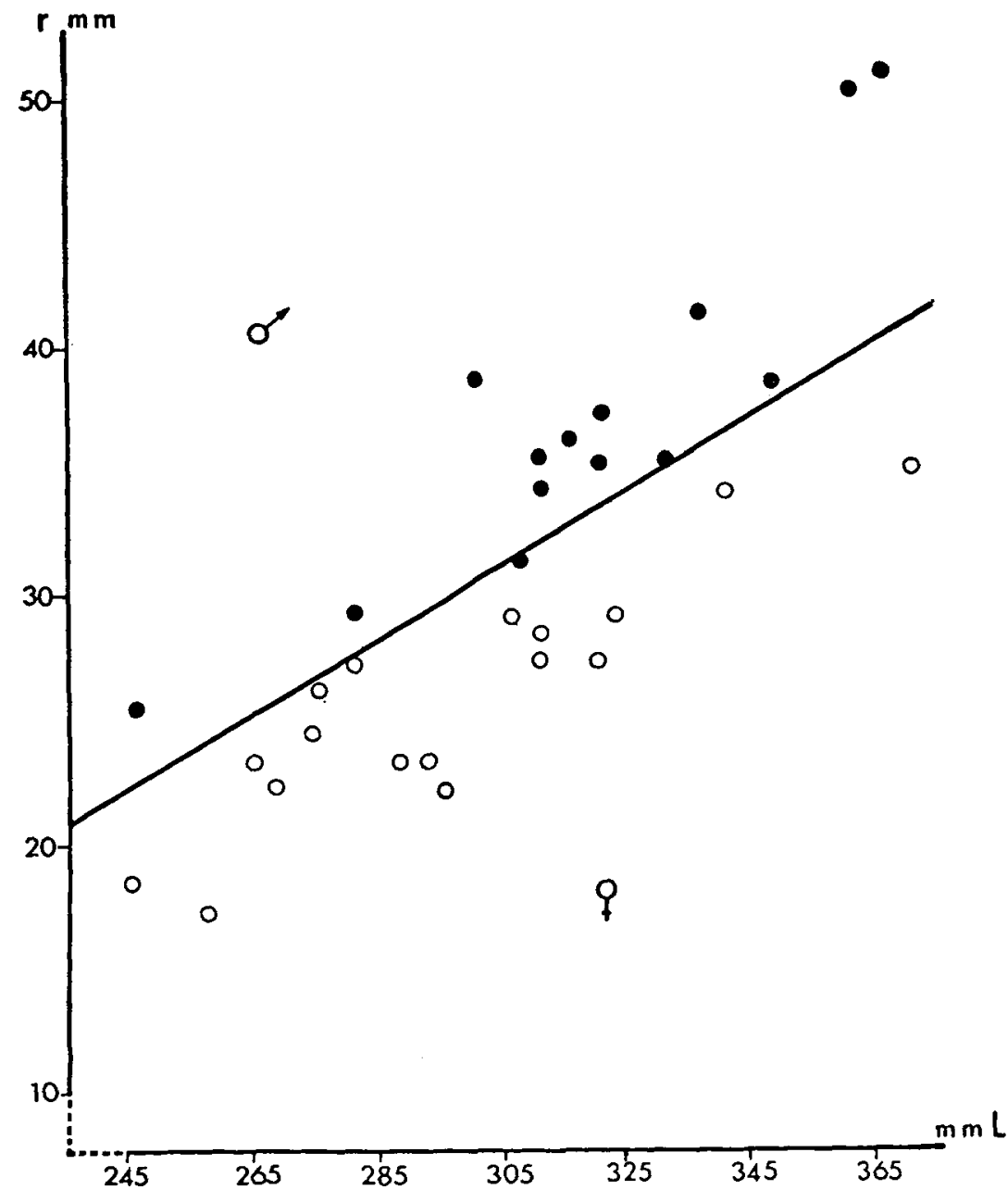

Figure 6 - Détermination du sexe de l'Ombre commun par comparaison de la longueur de l'antépénultième rayon de la dorsale $(r)$ en fonction de la longueur totale du poisson (L).

On peut en conclure que le potentiel de la fraction mature des Ombres de deux ans est en grande partie annihilé durant l'automne précédant la première ponte.

Dans le cas de l'Ance, où la croissance est beaucoup plus lente, les Ombres sont beaucoup mieux protégés à l'automne de leur deuxième année, d'autant plus que la fermeture de la pêche, qui s'effectue en décembre sur la rivière d'Ain, a lieu dès la fin de septembre dans l'Ance, classée en rivière à Truite de première catégorie. Cependant les Ombres de l'Ance n'atteignent probablemient pas la maturité au bout de cette deuxième année, et ne sont plus protégés lors de leur troisième été.

- En élevant la taille de capture à $30 \mathrm{~cm}$, on obtiendrait sur l'Ain la protection d'environ la moitié des Ombres de deux ans puisque la taille moyenne 
à la fin de la deuxième année est de $29,5 \mathrm{~cm}$. Cependant il s'agirait principalement d'immatures car ce sont surtout parmi les Ombres de plus de $30 \mathrm{~cm}$ que semblent se rencontrer les femelles adultes.

L'effet d'une telle mesure ne serait donc pas aussi efficace qu'on pourrait l'espérer. Elle permettrait cependant à une proportion plus importante de grands poissons de deux ans de franchir ce cap difficile car ils ne seraient susceptibles d'être pêchés que pendant un laps de temps nettement plus court.

Cette proportion serait plus grande dans la Loue où la croissance est légèrement plus faible, mais la maturité chez les poissons de deux ans doit être en conséquence nettement plus rare.

Dans l'Ance, les Ombres de cet âge seraient, quant à eux, totalement protégés, mais ceux de trois ans ne le seraient que très partiellement.

- Une taille légale de $34 \mathrm{~cm}$ permettrait sur l'Ain de protéger pratiquement toute la génération de deux ans (97,5\% des individus), d'où probablement une efficacité appréciable dans la reproduction des individus les plus développés. Un autre avantage serait de protéger également une proportion non négligeable (environ $20 \%$ ) des Ombres âgés de trois ans, ceux ayant grandi le plus lentement mais qui sont à cet âge-là tous reproducteurs, et d'après la littérature, certainement plus féconds que ceux de deux ans.

Cette même taille de $34 \mathrm{~cm}$ protègerait,jusqu'à leur troisième année révolue à la fin de laquelle a lieu sans doute la première reproduction efficace, presque tous les Ombres de la Loue et la totalité des Ombres de l'Ance.

La conséquence d'une telle élévation de la taille minimale de capture serait en réalité, pour les pêcheurs de l'Ain, la perte de la première demi-saison d'automne suivant la mise en application de cette disposition. Ils ne pourraient alors pas prendre les poissons de deux ans. Ils seraient ainsi amenés à rejeter $90 \%$ des captures faites habituellement en automne, mais ils retrouveraient, dès le début de la saison suivante et durant toute celle-ci, ces mêmes poissons à des tailles supérieures à $34 \mathrm{~cm}$ et donc beaucoup plus attrayantes.

II n'en résulterait donc qu'une baisse du rendement de la pêche lors du premier automne, compensée dès le printemps suivant par un gain appréciable de taille des poissons pêchés et, dans les deux années suivantes, par un accroissement de leur nombre grâce à un meilleur taux de reproduction.

L'Ombre commun subit actuellement une forte régression, consécutive au développement économique et parallèle à celle observée aux Etats-Unis oủ l'Ombre du Michigan a complètement disparu, et celui du Montana ne survit plus. que dans un seul cours d'eau.

Cette régression ne semble pas pouvoir être compensée efficacement en France par des introductions dans de nouveaux bassins.

II est donc indispensable de maintenir les habitats actuels de ce poisson dans leurs caractéristiques naturelles de pureté, et de leur éviter toute pollution ou remaniements. Certaines rivières mériteraient de bénéficier, à ce titre, d'une protection efficace et d'être classées comme sites naturels d'intérêt national.

L'attrait touristique constitué par les rivières à Ombre, qui sont, comme la présence même de ce poisson l'atteste, les rivières encore pures, est un fait économique considérable dont l'importance est appelée à s'accroitre dans l'avenir. Indépendamment de la valeur marchande des poissons pêchés (des milliers d'Ombres, de Truites et d'autres espèces chaque année dans la basse 
vallée de l'Ain), le nombre de journées de loisir passées chaque année au bord de ces rivières (plus d'un milliọn pour l'Ain) joue un rôle social et économique que nulle autre activité ne peut remplacer.

Aucune rivière française n'est actuellement à l'abri de dégradations d'une sorte ou d'une autre, entreprises pour des motifs pas toujours valables, et tous les peuplements d'Ombres sont menacés. Seule une volonté commune des autorités et des pêcheurs peut permettre la survie de cette espèce dans notre pays.

Une des premières mesures à prendre serait l'augmentation de sa taille légale de capture. Cette décision sera sans doute impopulaire auprès des pêcheurs qui peuvent s'estimer à juste titre beaucoup moins fautifs que les pollueurs ou les aménageurs dont les grands projets exigent la dénaturation des rivières (par exemple la rectification de la Loue en aval de Parcey). Elle ne devrait pourtant pas léser les pêcheurs puisqu'elle augmenterait le nombre de poissons en assurant une meilleure reproduction. Il est bien évident que si elle n'est pas accompagnée de dispositions précises pour la protection des eaux, cette modification de la taille légale de capture ne fera que retarder l'échéance.

Cette espèce est pourtant un de nos poissons d'eau douce les plus beaux et les plus recherchés et elle possède l'avantage, ce travail le confirme, d'être probablement un des plus productifs.

\section{BIBLIOGRAPHIE SOMMAIRE}

BANARESCU P., 1964. Pisces Osteichthyes, Bucuresti, Fauna R.P.R., 13, 959 p.

DAHL J., 1962. Studies on the biology of Danish stream fishes. I. The food of the Grayling (Thymallus thymallus L.) in some Jutland streams. Meddr. Danm.
Fisk. -og Havunders, 3, 199-264.

ERIKSEN C., 1975. Physiological ecology and management of the rare "southern * Grayling Thymallus arcticus tricolor Cope. Verh. Int. Verein. Limnol., 19, 1593-1598.

FOISSAC M., 1974. Contribution à l'étude de l'acclimatation de l'Ombre commun (Thymallus thymallus) dans la région Midi-Pyrénées. Thèse spécialité, Toulouse.

HUTTON J.A., 1923. Something about Grayling scales. Salm. Trout Mag., 1, 3-8.

JANKOVIC D., 1960. Sistematika i ekologija lipljena jugoslavije. Beograd, 144 p.

MiCHA J.C., 1971. Densité de population, âge et croissance du Barbeau Barbus barbus L. et de l'Ombre Thymallus thymallus L. dans l'Ourthe. Annls Hydrobiol., 2, 1, 47-68.

OLIVA O., 1974. On the systematics of the European Grayling, Thymallus thymallus (Linnaeus 1758) (Osteichthyes Thymallidae). Vest. csl. Spol. Zool., 38, 3 . 187-214.

PETERSON H.H., 1968 The Grayling, Thymallus thymallus (L.) of the Sundsvall bay area. Inst. Freshwat. Res. Drottningholm, 48, 36-56.

SVETINA M., 1961: L'Ombre commun et son élevage. Zoo, Antwerp, 27, 49-55. 